\title{
MICRO ROBOTS CONTROLADOS POR ACTUADORES ELECTROMAGNÉTICOS EN APLICACIONES MÉDICAS
}

\section{MICRO ROBOTS CONTROLLED BY ELECTROMAGNETIC ACTUATORS IN MEDICAL APPLICATIONS}

\author{
PhD. Hernando León-Rodríguez, Ing. Daniel Murcia Rivera
}

Universidad Militar Nueva Granada, Facultad de ingeniería, Programa de Ingeniería Industrial, Grupo de Investigación en Producción, Innovación Tecnología. Carrera 11 N 101 - 80, Bogotá Cundinamarca, Colombia. +57 1650000.

E-mail: \{hernando.leon, u1802440 \}@Unimilitar.edu.co.

\begin{abstract}
Resumen: Los robots para aplicaciones médicas en la micro escala son la combinación de la tecnología de fabricación tradicional con tecnología de MEMS (micro electromechanical systems) sumadas con tecnologías como nano-tecnología, ingeniería biomédica y ciencia de los Materiales. Los robots en escala humana aplicados a la medicina son dispositivos desarrollados y adaptados con tecnologías de aplicaciones industriales. Sin embargo, en la actualidad la ciencia médica busca miniaturización para minimizar las cirugías, el dolor y el sangrado en el paciente; es aquí donde los micros robots son una tecnología emergente que permitirá llevar con micro-cámaras, micromanipuladores, y nano-sensores a áreas del cuerpo que no requerirán cirugía sino la utilización de los orificios naturales del cuerpo. Este artículo presenta una revisión de los dispositivos robóticos médicos del presente y del futuro cercano en la salud.
\end{abstract}

Palabras clave: Microrobots, cirugía de mínima invasión, actuador electromagnético, bobinas de Helmholtz y Maxwell, región de interés.

\begin{abstract}
Robots for medical applications at the micro scale are the mixture of traditional manufacturing technology with MEMS technology (micro-electro-mechanical systems) combined with technologies such as Nano-Technology, Biomedical Engineering and Materials Science. Robots at human scale applied into medicine are devices adapted with technologies from industrial applications. Furthermore, medical science currently seeks miniaturization with the aim of minimize surgeries, pain and bleeding on patients; these targets are an emerging technology where micros robots can carry onboard microcameras, micro-manipulators, and Nano-Sensors to be able to go into human body that will not require surgery, just by using the body's natural orifices. This article presents a brief review of medical robot at the macro and micro scale in the near future..
\end{abstract}

Keywords: Microrobots, Minimal invasion surgery, Electromagnetic actuator, Helmholtz and Maxwell coils, Region of interest.

\section{INTRODUCCIÓN}

Hoy en día, los robots médicos son los cirujanos no humanos de alto rendimiento, con una precisión inigualable y la capacidad de trabajar sin fatiga.
Estos robots son ampliamente utilizados en diversas prácticas médicas, incluyendo procedimientos quirúrgicos de gran dificultad y velocidad, donde han revolucionado completamente la eficiencia y la calidad de los 
servicios de atención de salud en varias partes del mundo.

El primer robot médico conocido utilizado por la industria médica fue en 1985, cuando el robot PUMA 560 colocó una aguja para una biopsia cerebral utilizando la guía CT (Computed tomography) (Davies, 2000). Años más tarde, el PROBOT de Imperial College de Londres ayudó a los médicos a realizar cirugía de próstata en un paciente. (Harris et al., 1997) Desde entonces, se han realizado estudios persistentes sobre sistemas robóticos para uso específico en el cuidado de la salud por parte de investigadores médicos.

Uno de los robots más famosos es el sistema quirúrgico Da-Vinci; (Tewari et al., 2002) este utiliza un método de cirugía mínimamente invasivo (MIS) frecuentemente maniobrado para reparación de válvulas cardíacas, prostatectomías, remoción de tumores abdominales y procedimientos ginecológicos. (Tewari et al., 2002) El cirujano maniobra los brazos robóticos del sistema mientras mira una pequeña cámara endoscópica de alta resolución es insertada a través de una incisión. La capacidad articulada de la muñeca del robot supera la amplitud de movimiento de la mano humana, permitiendo el movimiento en un espacio mucho más pequeño. Esto genera en el paciente incisiones más pequeñas, menos sangre, dolor y trauma y un tiempo de curación más rápido que la cirugía regular.

Varios robots médicos se utilizan en una amplia gama de aplicaciones quirúrgicas, desde la neurocirugía a la radiocirugía, incluso en rehabilitación, pediatría y ortopedia. En Israel, un robot miniatura llamado ViRob fue desarrollado con el fin de arrastrarse a través del cuerpo humano, localizar un tumor, y tratarlo con medicamentos. Su tamaño era solamente de un milímetro de largo y cuatro milímetros de extremo a extremo, el Vibot utiliza pequeños brazos controlados por un campo electromagnético para propulsar su camino a través de diferentes cavidades del cuerpo humano, en busca de tumores mortales para ser tratados. (Microbotmedical; 2017)

En muchas partes del mundo, los hospitales utilizan robots para tareas que requieren precisión y rapidez, como la entrega y recuperación de suministros y la mezcla y dispensación de medicamentos. En Europa y los EE.UU., un robot se utiliza para mezclar y medir los medicamentos, específicamente los utilizados en la quimioterapia.
(Ham et al., 2015) Los errores humanos en este proceso pueden ser mortales, ya que el menor error en la mezcla podría poner en peligro tanto a los pacientes como al personal del hospital. El uso de un robot así elimina la necesidad de cálculo manual y reduce en gran medida la posibilidad de error humano.

Otros robots médicos patrullan hospitales como enfermeras y miembros del personal. En un centro médico de Maryland, un robot llamado Gower navega por los pasillos, usa el servicio de ascensores, abre las puertas, entrega los medicamentos de los pacientes en la estación de enfermeras y ayuda a entregar los medicamentos a sus pacientes más rápido y en forma autónoma. (Pandey et al., 2012) En los hospitales de Asia, los pacientes en la unidad de cuidados intensivos son visitados regularmente por robots de ruedas con monitores de computadora para la cabeza (avatars). Estos robots son controlados de forma remota por médicos desde un centro de mando, lo que permite a los médicos verificar simultáneamente a sus pacientes y leer sus signos vitales sin tener que abandonar su puesto. En las áreas de EE.UU. con escasa cobertura de especialistas, la telemedicina que utiliza la misma tecnología permite a especialistas remotos hacer los procedimientos iniciales de evaluación del paciente como la gestión del accidente cerebrovascular y/o dar diagnósticos efectivos. Usando una cámara de vídeo de un robot, un neurocirujano puede observar y hablar con los pacientes y revisar sus tomografías computarizadas y otros resultados de laboratorio, incluso si están a cientos de kilómetros de distancia. (Nathoo et al., 2005).

Por otro lado Los robots son cada vez más populares en las farmacias. El McKesson ROBOTRxm, por ejemplo, almacena y dispensa dosis únicas de medicamentos para hospitales enteros. Los farmaceutas simplemente introducen recetas en una computadora, mientras que el robot recolecta la dosis escaneanda y los códigos de barras de los medicamentos. Finalmente, el robot saca las medicinas y realiza un seguimiento de todo para asegurar que el medicamento correcto llegue al paciente adecuado. (McKesson, 2018)

Otro dispositivo similar es el IntelliFill iv, un robot que puede llenar hasta 60 jeringas de fármacos específicos para el paciente cada hora. También permite asegurar que el medicamento correcto en la dosis adecuada llegue al paciente adecuado. (Osborne, 2017) Esto ha llevado a los hospitales a computarizar los sistemas para órdenes de 
prescripción y utilizar dispositivos que automatizan el proceso de llenado de la prescripción médica. (Inguanti, 2016) Claramente, aunque el elemento del tacto humano es vital en la industria médica, algunas áreas parecen mejor ser dejadas a las máquinas. El objetivo de estos robots, después de todo, es mejorar la salud y la vida de los seres humanos.

\section{ROBÓTICA MÉDICA A LA MACRO- ESCALA}

Los robots a la macro escala utilizados en medicina principalmente están enfocados en el desarrollo de intervenciones quirúrgicas, como ejemplo: cirugía cardiaca (Falk et al., 2000), gastro-intestinal (Moreno et al., 2008) o de neurocirugía (Karas et al., 2007). Esto dispositivos permiten al cirujano realizar el procedimiento de una manera más precisa minimizando el dolor y la recuperación del paciente; son llamadas MIS (minimal invasion surgery) o cirugías mínimamente invasivas (Castillo, 2013) (ver figura 1).
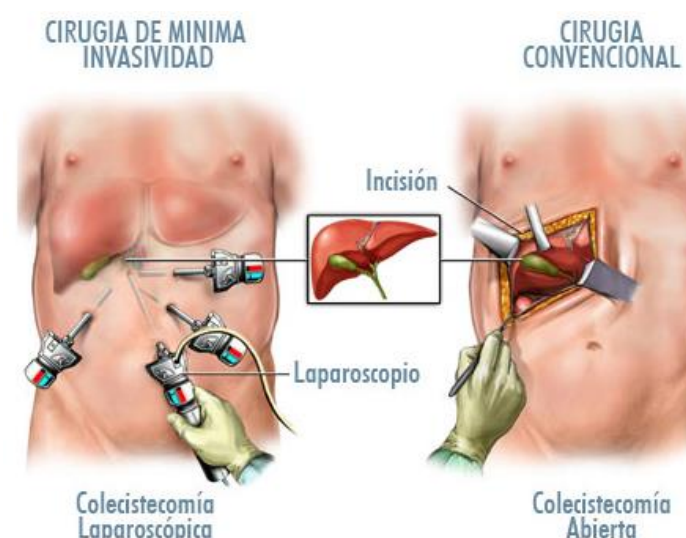

Fig. 1. Cirugía mínimamente invasiva vs cirugía tradicional. (Castillo, 2013)

Este tipo de cirugía MIS permite que en la practica el paciente tenga menos riesgo de infección en la herida, menos tiempo en quirófano y un tiempo de recuperación más corto; además, de las ventajas estéticas. Sin embargo el cirujano pierde el contacto directo tanto visual como táctil durante la operación y se hace necesario un riguroso aprendizaje de las técnicas quirúrgicas con los sistemas robóticos.

Entre las técnicas para minimizar el error tecnológico en los sistemas tele-quirúrgicos de MIS, el cirujano practica con antelación los movimientos que serán ejecutados por el robots; además de usar alternativas como sistema de control compartido donde uno o varios cirujanos ejecutan movimientos estables mientras el otro cirujano controla todo el proceso.

El dispositivo robótico quirúrgico usado en los hospitales en la actualidad es el sistema Davinci (ver figura 2); fue diseñado y fabricado por la empresa norteamericana Intuitive Surgical (Davinci, 2018), aprobado por la FDA (Food and Drugs Administration) en el año 2000 (Densford, 2017). Tiene el objetivo de potenciar las capacidades y habilidades del cirujano y consta de un robot articulado de 4 DOF que trabaja en el paciente llevando en sus brazos una cámara de alta definición y los instrumentos de trabajo de MIS como son: pinzas, inyectores, succión, tijeras, biopsias entre otras.

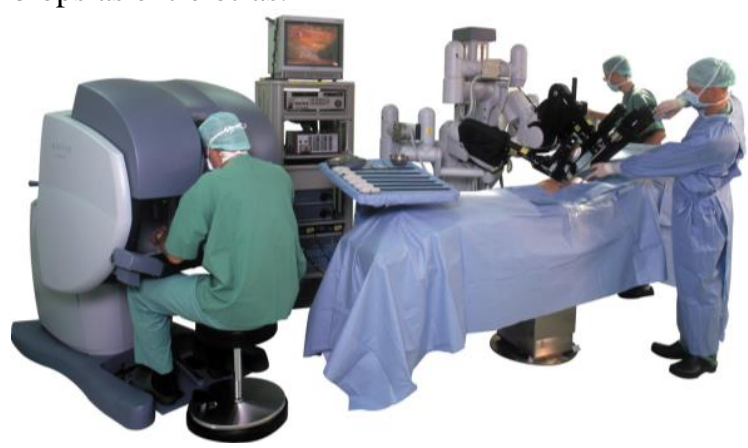

Fig. 2. sistema robótico de cirugía Davinci

(Davinci, 2018)

La otra parte del sistema DaVinci es la consola donde el cirujano controla todos los movimientos de cada una de las pinzas y con ayuda de imagen tridimensional puede ampliar el campo de visión al interior del cuerpo. El sistema recibe las órdenes en tiempo real, reproduciendo los movimientos de la mano, la muñeca, pies, dedos del cirujano y en la actualidad está en desarrollo el movimiento de los parpados. (Davinci, 2018)

\section{ROBÓTICA MÉDICA A LA MICRO- ESCALA}

La física a escala pequeña puede volverse un poco extraña en comparación con la macro escala debido a la aparición de fuerzas y efectos que generalmente son despreciados a macro escala. Los micro robots son sistemas que lidian con este tipo de fuerzas, y para gran parte de las aplicaciones, se requiere el control de la posición de estos. Por ello, se desarrolló un prototipo de un micro robot relativamente grande con un actuador electromagnético.

La micro-robótica es una tecnología emergente en la última década; está inspirada en la locomoción 
biológica de organismos a la micro escala. (Kim et al., 2017) la micro-robótica se ha enfocado principalmente en soluciones de aplicación médica, específicamente por ejemplo: minimizar el tamaño de la cirugía (minimal Invasion Surgery) (Nelson et al., 2010) (Fusco et al., 2014), entrega de medicina en órganos específicos del cuerpo (drug delivery system) (Nguyen et al., 2016) (Yu et al., 2010), navegación por el aparato circulatorio (Park et al., 2010) (Nagy et al., 2008) y/o digestivo (Donald et al., 2010), entre otros.

Debido a la necesidad de miniaturización de los componentes y los desafíos asociados a la fabricación de los sistemas robóticos en la micro escala, su construcción se fundamente en la tecnología MEMS. (Wautelet et al., 2001) Pero esto significa que los sistemas micro-robóticos carecen de la complejidad de sus equivalentes en la escala convencional. Las leyes físicas que predominan en la micro escala son principalmente las fuerzas superficiales (Purcell, 1976) (Abbott et al., 2007); en tal razón, el almacenamiento de energía que principalmente está basado en el volumen ejemplo: las baterías, es menos eficaz, dando como resultado que la mayoría de los sistemas micro-robóticos emplean suministro de potencia externo. (Pawashe et al., 2009)

Entre los mecanismos de actuación más comunes está el accionamiento electrostático (Go et al., 2014), sistema electromagnético (Villangca et al., 2016), laser control (Fumihito et al., 2006) (Peyer et al., 2013), propulsión biológica basada en bacterias (Zhang et al., 2009) (Yesin et al., 2005), entre otros métodos prometedores de futuro. En el caso de los sistemas electromagnéticos, presentado a continuación, existen diversos tipos de configuración de actuadores, entre los más recocidos están: los pares de bobinas circulares de Helmholtz y Maxwell (Cugat et al., 2003) (Kummer et al., 2010), las bobinas concentradas o campo magnético no-uniforme (OctoMag) (Diller et al., 2016) (Jeong et al., 2012) y las bobinas modificadas geométricamente para ampliar la región de interés (Saddle Coils) (Choi, 2010) (Park et al., 2013).

La aplicación más destacada de estos pequeños sistemas, son los relacionados con la salud. Los micro robots pueden tratar los vasos bloqueados debido a una mala alimentación y actividad física deficiente (Park et al., 2010). También pueden administrar medicamentos en lugares específicos, aumentando la efectividad del medicamento y reduciendo los efectos secundarios en otras partes del cuerpo (Nelson et al., 2010). La endoscopia es otra área de aplicación, en (Yim et al., 2012) y (Ciuti et al., 2009) se desarrollan sistemas electromagnéticos para micro robots endoscópicos especializados. Comparando con los métodos tradicionales, esta tecnología puede superar los problemas de infección debido al entorno (Cowen , 2001) y los riesgos debido a la competencia del médico (Romagnuolo et al., 2011).

\subsection{Sistema actuador electromagnético para micro-robots}

El sistema actuador consiste en 3 pares de bobinas electromagnéticas alineadas en los 3 ejes cartesianos. Estos pares de bobinas son perpendiculares y estacionarias, compuestas por 3 bobinas Helmholtz y 3 bobinas Maxwell, que permiten reproducir movimientos en el micro-robot de 6 grados de libertad. (Ver figura 3)

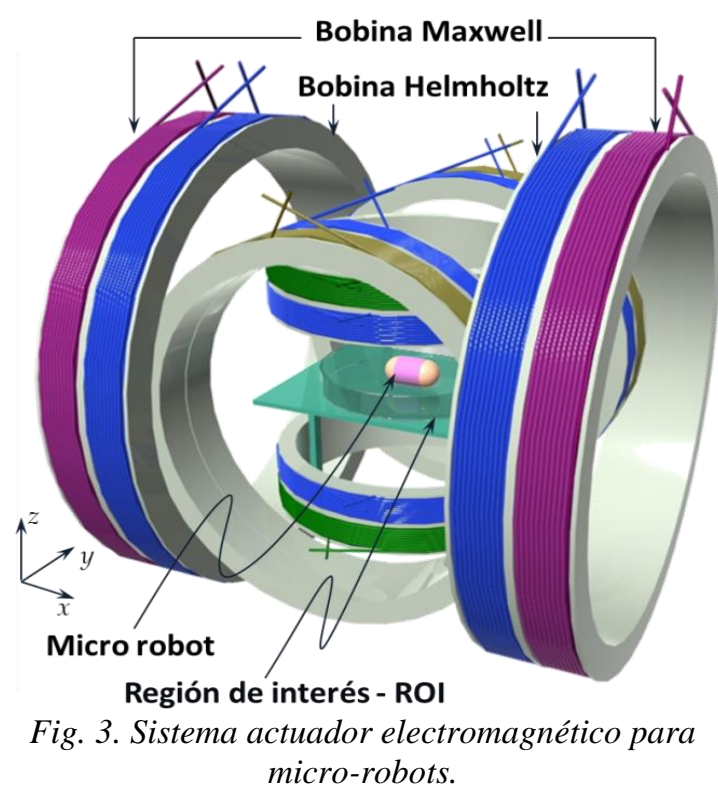

Esencialmente, la bobina Helmholtz al energizarse produce un flujo magnético uniforme en su parte intermedia o región de interés (ver figura 4: arribaizquierda), esto permite que al colocar un microrobot se pueda alinear en dirección y orientación producida por el campo magnético externo (ver figura 4: abajo-izquierda). Por otro lado, la bobina de Maxwell permite generar un flujo magnético gradiente-uniforme que al combinarse con la bobina de Helmholtz permitirá producir la fuerza de propulsión del micro robot. (Ver figura 4: derecha-abajo). 

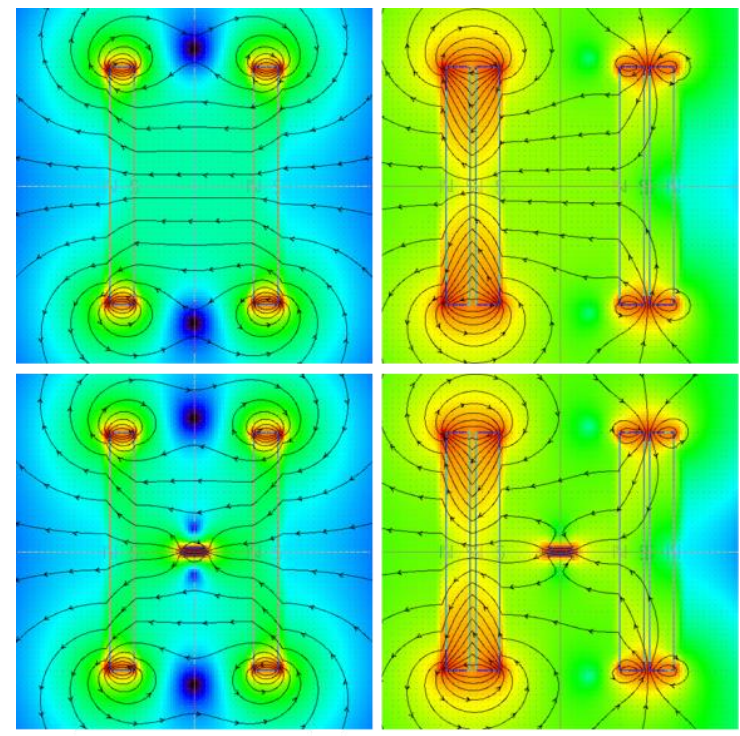

Bobina Helmholtz

Bobinas Helmholtz \& Maxwell Fig. 4. Simulación del comportamiento individual y combinado de las bobinas Helmholtz y Maxwell.

(Vizimag software).

Fundamentalmente y analizando el comportamiento del actuador electromagnéticos (EMA) en forma bidimensional (plano $x-y$ ); el diagrama esquemático de la figura 3 nos muestra la configuración en los 3 ejes cartesianos de los diferentes pares de bobinas del sistema actuador. La figura 5 nos muestra las bobinas $H x_{l^{-}} H x_{2}$ y $H y_{1^{-}}$ $\mathrm{Hy}_{2}$ representadas en el diagrama como bobina Helmholtz en los ejes y cuadrantes cartesianos positivo y negativo. Adicionalmente las bobinas $M x_{1}-M x_{2}$ y $M y_{1}-M y_{2}$ correspondientes a las bobinas Maxwell de igual configuración en ejes y cuadrantes. Finalmente, la figura 3 muestra la dirección de la corriente en cada bobina; donde al ser controlada independientemente permite el direccionamiento del campo magnético en la trayectoria del eje $x$ y el eje $y$.

La Bobina de Helmholtz consiste en el arrollamiento de 2 bobinas independientes con iguales condiciones geométricas, diámetro del alambre y numero de vueltas. Estas bobinas a ser colocadas en forma paralela producen un flujo magnético uniforme (figura 4 derecha-arriba). La distancia teórica $\mathrm{d}$ entre los dos arrollamientos para producir el flujo magnético uniforme ideal debe ser igual al radio $\mathrm{r}$ de las bobinas $H y_{1}$ y $H y_{2}$ o $H x_{1}$ y $H x_{2}$. (ver figura 5). En conclusión, la bobina Helmholtz cumple con la relación: $d=r$. Para el caso de la corriente que fluye a través de la bobinas, esta debe tener la misma dirección y la misma intensidad; dando como resultado las líneas de campo magnético uniforme en medio de los dos arrollamientos. Este flujo magnético es definido como un vector que al ser controlado en dos pares de bobinas Helmholtz, permite obtener cualquier dirección de orientación en el plano $x-y$ que será reproducido por el micro-robot.
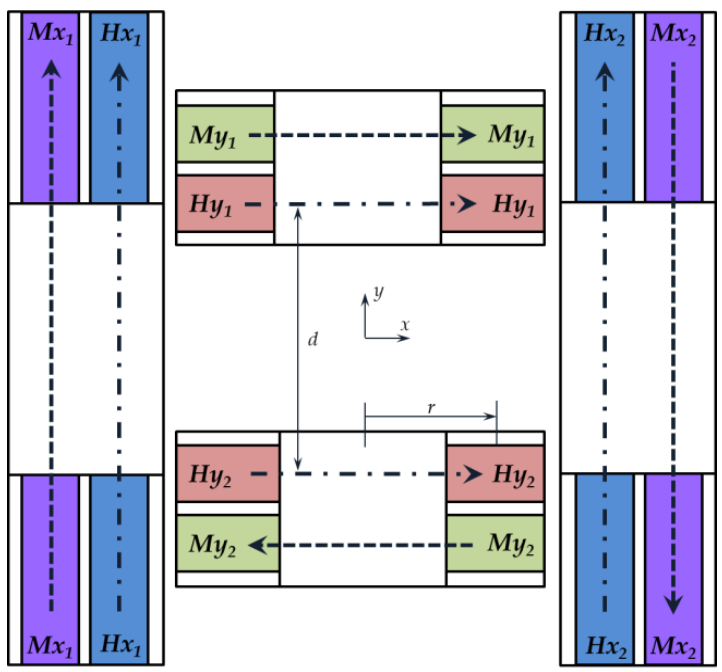

Fig. 5. Diagrama esquemático del sistema actuador electromagnético (EMA) plano $x-y$.

En forma similar la bobina de Maxwell se compone de dos arrollamientos que cumplan con las mismas condiciones de geometría, diámetro del alambre y numero de vueltas. Estos dos arrollamientos al ser colocados paralelamente deben cumplir con la relación $d=\sqrt{3} r$. Dónde: $d$ es la distancia entre los dos arrollamientos y $r$ el radio del solenoide. Adicionalmente, en la bobina de Maxwell la corriente aplicada a los dos solenoides debe ser la misma intensidad, pero la dirección del flujo de corriente debe ser opuesta. La densidad del flujo magnético producido por la bobina Maxwell es gradiente-uniforme generado a los largo de los ejes $x, y$. Este flujo magnético también se define en forma vectorial y es quien produce la impulsión del micro robot.

El diseño mecánico del actuador electromagnético es acondicionado para obtener el mayor espacio y posición de la región de interés (ROI), donde los diámetros de los pares de bobinas Helmholtz y Maxwell son diferentes debido a la restricción geométrica y posición de cada bobina, pero ambas satisfacen la definición teórica. Finalmente, para obtener el mismo flujo magnético de las bobinas Helmholtz y Maxwell en los ejes $\mathrm{x}, \mathrm{y}, \mathrm{z}$ esto es posible mediante dos alternativas: la primera opción es mantener constante la corriente eléctrica y calcular el número de vueltas y diámetro del alambre de las bobinas. La segunda, es mantener un diámetro constante en el espesor del alambre, 
obteniendo, un número de vueltas definido para cada par de bobinas; resultando, en la variación y control de la corriente eléctrica en cada par de bobinas. Este último fue aplicado para este actuador (ver figura 6).

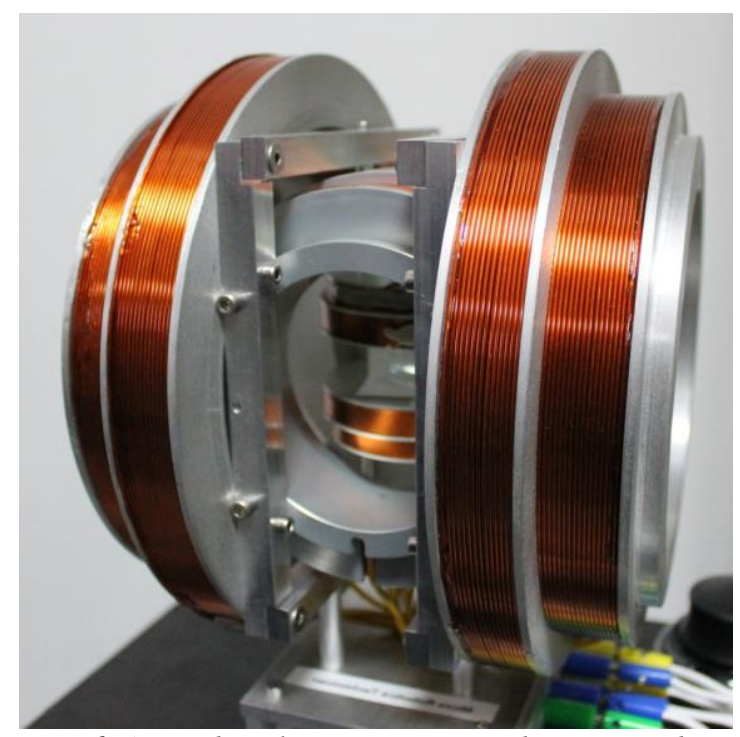

Fig. 6. Actuador electromagnético de micro-robots

Las ecuaciones (1) y (2), presentadas por (28), rigen la forma en que un campo magnético de intensidad $\vec{H}$ influye sobre la dinámica de un imán permanente (micro-robot).

$$
\begin{gathered}
\vec{F}=\mu_{0} V(\vec{M} \times \nabla) \vec{H} \\
\vec{T}=\mu_{0} V \vec{M} \times \vec{H}
\end{gathered}
$$

Reescribiendo (1) la ecuación para un modelo en 3 dimensiones se expresa como:

$$
\vec{F}=\mu_{0} V\left(M_{x} \frac{\partial \vec{H}}{\partial x}+M_{y} \frac{\partial \vec{H}}{\partial y}+M_{z} \frac{\partial \vec{H}}{\partial z}\right)
$$

Al usar el principio de superposición, el campo magnético $\mathrm{H}$ total es la suma del campo producido por las bobinas de Maxwell $\overrightarrow{H_{m}}$ y la bobina Helmholtz $\overrightarrow{H_{h}}$.

$$
\vec{H}=\overrightarrow{H_{m}}+\overrightarrow{H_{h}}
$$

\subsection{Resultado y experimentación control manual}

Desde la teoría y la simulación numérica, se confirmó que la dirección del micro-robot está regulada por las dos bobinas Helmholtz y el movimiento del micro-robot por los dos pares de bobinas de Maxwell. En modo de control manual el campo magnético es accionado para orientar el micro robots en la dirección deseada (ver figura 7).

La primera prueba se realiza controlando la corriente de la bobina Helmholtz, donde la magnitud y la dirección del campo magnético fueron reguladas arbitrariamente. Por lo tanto, la dirección del campo magnético se modificó ajustando la relación del flujo de corriente para cada eje, donde el torque generado permite girar el micro-robot a la dirección deseada. La Figura 7 se muestra los resultados experimentales de la rotación del micro-robot en 2 ejes. A través de estos experimentos, se validó que el micro-robot podría estar alineado en el las direcciones deseadas $\left(0^{\circ}, 45^{\circ}, 90^{\circ}, 180^{\circ}, 225^{\circ}\right.$ y $\left.270^{\circ}\right)$ por los dos pares de Bobinas de Helmholtz.
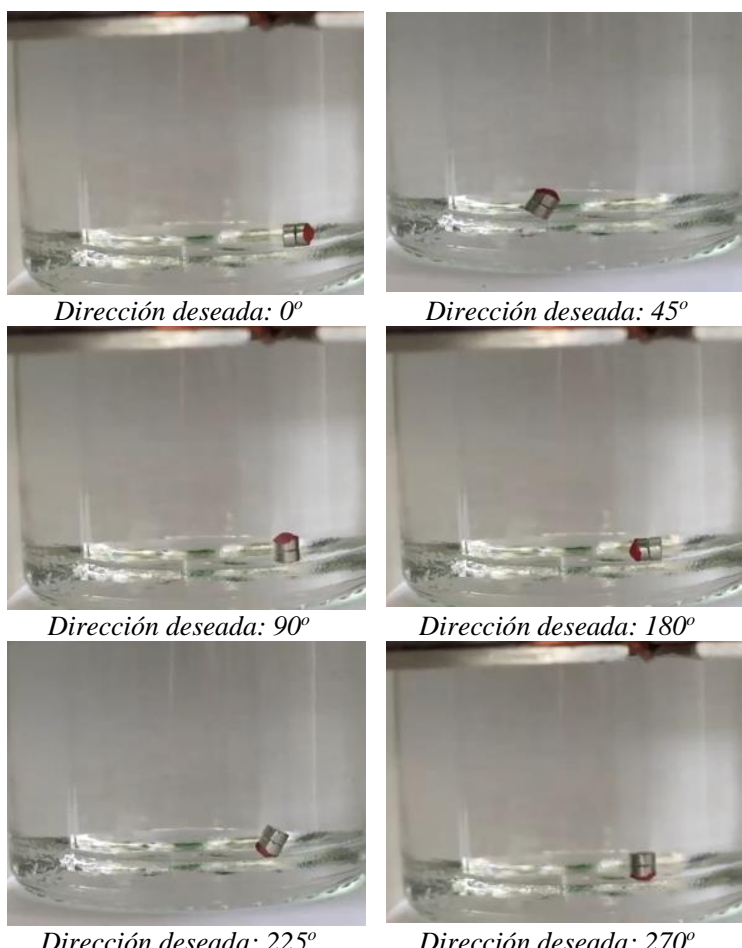

Fig. 7. Alineación del micro-robot bobinas Helmholtz, plano $x-z$

La segunda prueba es mediante la combinación de los pares de bobinas de Helmholtz y los pares de bobinas de Maxwell (ver figura 8) estos permiten generar la fuerza de propulsión en la dirección deseada del micro-robot. El micro-robot puede moverse en las 3 direcciones esperadas a lo largo de los 3 ejes cartesianos. En conclusión, el microrobot se alineó primero en la dirección deseada (figura 8; 90o eje z) por los dos pares de bobinas de Helmholtz y el desplazamiento lo genera los dos pares de bobinas de Maxwell resultando que el 
micro-robot se desplazó a lo largo de una trayectoria y dirección deseada.
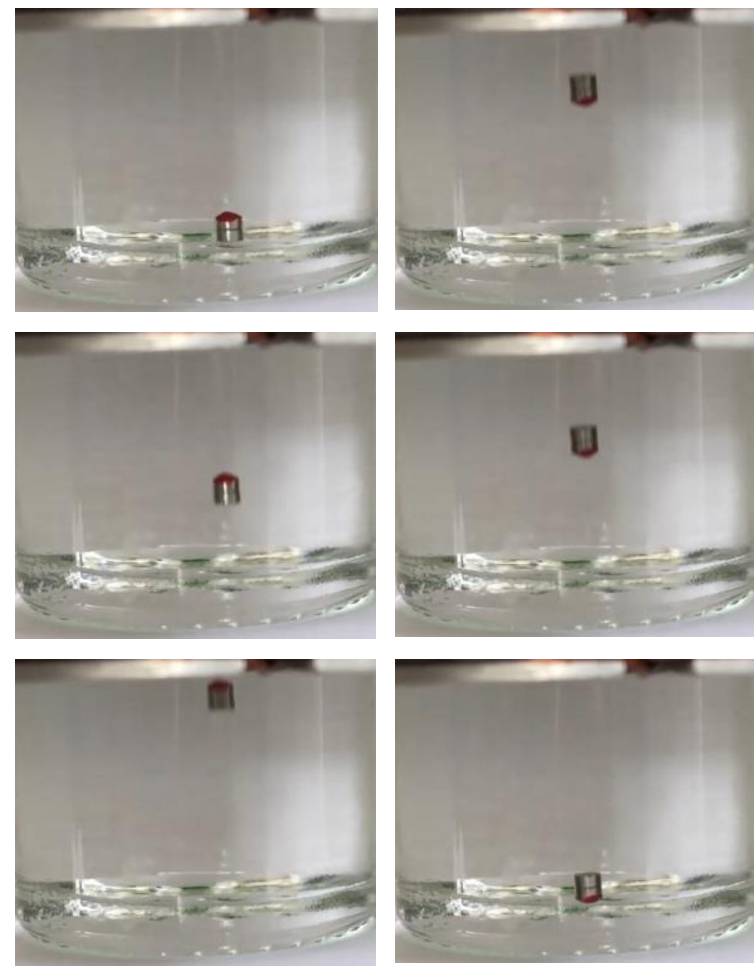

Eje z: desplazamiento positivo

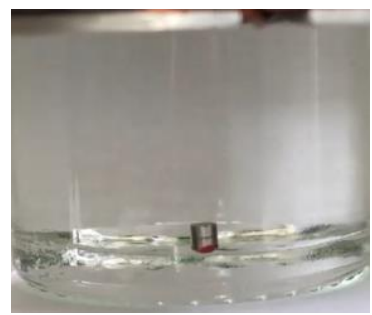

Eje z: desplazamiento negativo

Fig. 8. Desplazamiento del micro-robot bobinas Helmholtz y Maxwell, Eje: z, $90^{\circ}$

\subsection{Resultado y evidencia control autónomo}

La figura 9 muestra el sistema actuador del micro robots en forma autónoma, esto requiere de un sistema de procesamiento de imagen en tiempo real, el sistema de control de las bobinas de Helmholtz y Maxwell, las fuentes de poder y una unidad central de control de procesos.

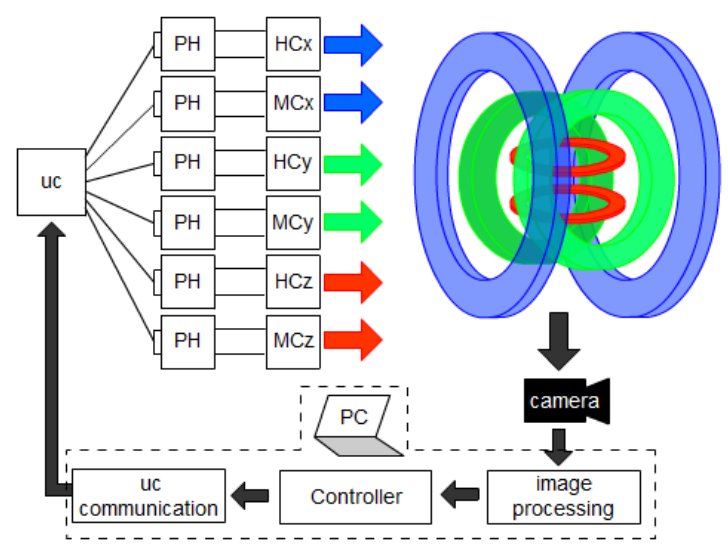

Fig. 9. Diagrama esquemático sistema actuador electromagnético; plano $x$-y; modo automático
La figura 10 muestras la trayectoria autónoma en forma de "S" del micro-robot en el plano $x$ - $y$. las gráficas a la derecha de la figura 10 nos muestra la posición actual del micro robot en cada eje cartesiano (x,y) $\rightarrow$ (rojo, verde); adicionalmente nos muestra la posición de referencia $(\mathrm{x}, \mathrm{y}) \rightarrow$ (amarillo, purpura), que es a posición a buscar en forma autónoma.
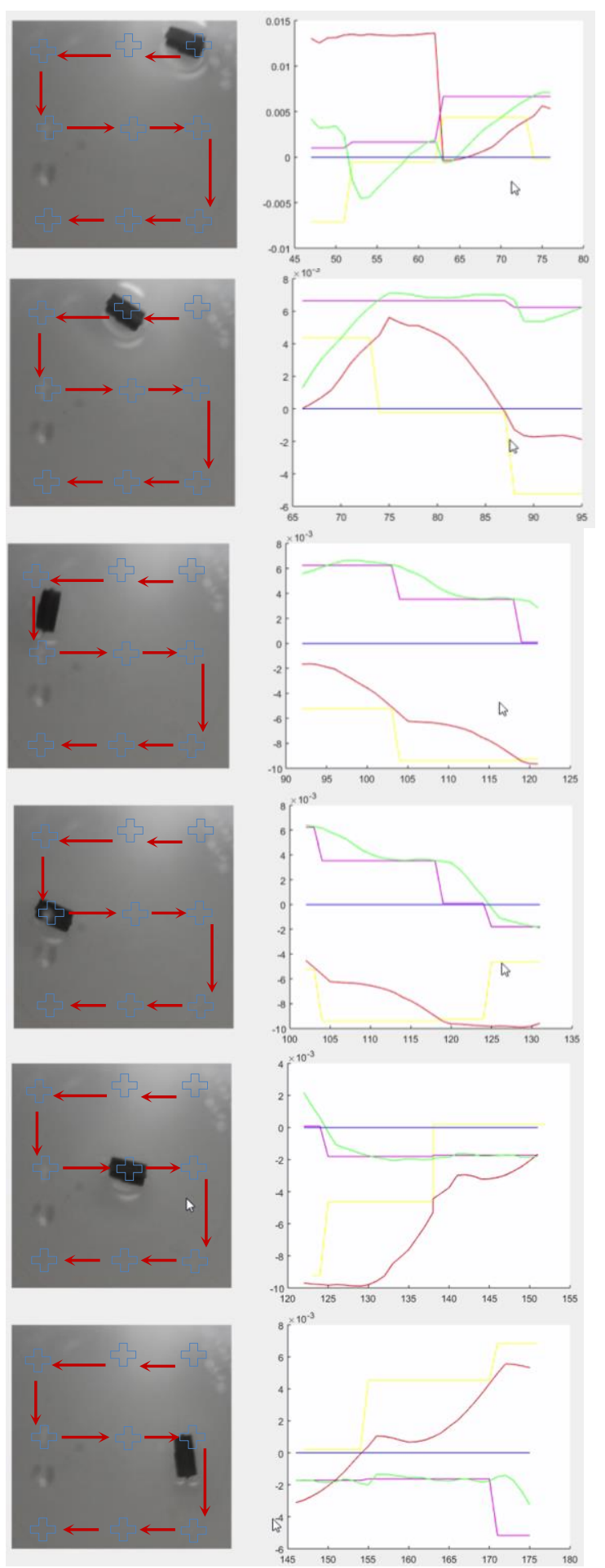


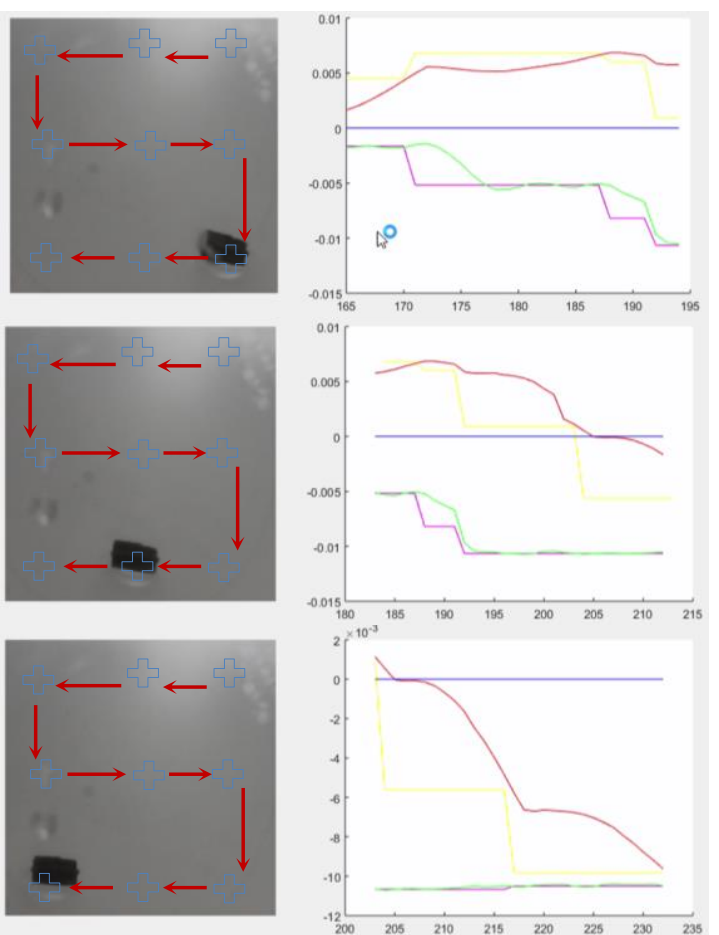

Fig. 10. Resultados de la trayectoria del micro robot en el plano $x$-y modo automático; Eje $y$ posición (m), Eje x-tiempo (s).

\section{CONCLUSIONES}

En el futuro, las cirugías y los dispositivos de aplicaciones médicas estarán asociados a los sistemas robóticos y serán parte de los procedimientos quirúrgicos. Por otro lado, la tecnología en micro robótica permitirá liderar parte de estos procesos de manipulación quirúrgica de mínima invasión o mediante la utilización de los orificios naturales buscando reducir el dolor en el paciente y reduciendo su tiempo de recuperación.

En la actualidad los sistemas electromagnéticos requieren de la compensación de gravedad correcta mientras se produce la rotación; en tal sentido, solo cuando la orientación deseada es alcanzada la compensación es correcta. Además, la influencia de Maxwell a veces causa desorientaciones altas que causan picos, más un error de cuantificación y la saturación hace que el seguimiento de trayectorias sea ruidoso. Esta es una razón para las pequeñas distorsiones presentes en la posición del robot.

Acerca del movimiento $x-y$ (donde la gravedad no está presente), emerge otro problema no lineal. La fricción entre el robot y las paredes, así como los cambios en la viscosidad por efectos de pared hacen que el observador no siga correctamente la posición real haciendo que el control se altere incluso cuando el robot está en el punto de flotación de la superficie del fluido.

\section{RECONOCIMIENTO}

Esta investigación fue soportada por la Universidad militar Nueva Granada, el proyecto Inv-Ing-2106.

\section{REFERENCIAS}

Abbott Jake J., Zoltán Nagy, Felix Beyeler, and Bradley J. Nelson (2007); Robotics in the Small; IEEE Robotics \& Automation Magazine.

Castillo cesar (2013), Cirugía de mínima invasividad; Disponible en: http://castillodelossantos.com; ; [Accedido: 20-may-2018]

Ciuti G., P. Valdastri, A. Menciassi and P. Dario, (2009); "Robotic magnetic steering and locomotion of capsule endoscope,".

Choi Hyunchul, Kyoungrae Cha, Jongho Choi, Semi Jeong, Seungmun Jeon, Gunhee Jang, Jong-oh Park, Sukho Park (2010); EMA system with gradient and uniform saddle coils for 3D locomotion of microrobot; Sensors and Actuators A $163,410-417$.

Cowen A. (2001), "The clinical risks of infection,".

Cugat Orphée, Jérôme Delamare, and Gilbert Reyne(2003); Magnetic Micro-Actuators and Systems (Magmas); IEEE Transactions on Magnetics, vol. 39,5 .

Davies B (2000); A review of robotics in surgery; Proc Instn Mech Engrs Vol 214 Part H; IMechE

Davinci Surgical System, Intuitive Surgical (2018); Disponible en:; https://www.intuitivesurgical.com; [Accedido: 20-may-2018]

Densford Fink (2017); Intuitive Surgical wins FDA not for daVinci $X$ platform; Disponible en: http://www.massdevice.com; [Accedido: 30-may2017]

Diller Eric, Joshua Giltinan, Guo Zhan Lum, Zhou Ye, and Metin Sitti (2016); Six-Degrees-of-Freedom Remote Actuation of Magnetic Microrobots; SAGE Journals, Vol 35, Issue 1-3.

Donald Bruce R., Christopher G. Levey, Igor Paprotny, and Daniela Rus (2010); Simultaneous Control of Multiple MEMS Microrobots; Springer-Verlag Berlin Heidelberg.

Falk Volkmar MD; Diegler, Anno MD, PhD; Walther, Thomas MD; Autschbach, Rudiger MD, PhD; 
Mohr, Friedrich W. MD, PhD (2000); Developments in robotic cardiac surgery; Current Opinion in Cardiology: November 2000 - Volume 15 - Issue 6 - pp 378-387.

Fumihito Arai, Toshiaki Endo, Ryuji Yamuchi, Toshio Fukuda (2006); 3D 6DOF Manipulation of Microobject Using Laser Trapped Microtool; Proceedings of the 2006 IEEE International Conference on Robotics and Automation.

Fusco Stefano, Franziska Ullrich, Juho Pokki, George Chatzipirpiridis, Berna Ozkale, Kartik M Sivaraman, Olgac, Ergeneman, Salvador Pane \& Bradley J Nelson; (2014); Microrobots: a new era in ocular drug delivery; Institute of Robotics and Intelligent Systems, ETH Zurich, Zurich, Switzerland.

Go Gwangjun, Hyunchul Choi, Semi Jeong, Cheong Lee, Bang Ju Park, Seong Young Ko, Jong-Oh Park, Sukho Park (2014); Position-based magnetic field control for an electromagnetic actuated microrobot system; Sensors and Actuators A 205, 215-223.

Ham Stephanie Lemmo, Ehsan Atefi, Darcy Fyffe, Hossein Tavana (2015); Robotic Production of Cancer Cell Spheroids with an Aqueous Two-phase System for Drug Testing; J. Vis. Exp. (98), e52754, doi: $10.3791 / 52754$.

Harris S J, F Arambula-Cosio, Q Mei, R D Hibberd, B L Davies, J E A Wickham, M S Nathan, B Kundu, (1997) The Probot - an active robot for prostate resection; Proceedings of the Institution of Mechanical Engineers, Part H: Journal of Engineering in Medicine Vol 211, Issue 4, pp. 317 -325 .

Inguanti Mary (2006); The IntelliFill iv for Health Technology; PP\&P.

Jeong Semi, Hyunchul Choi, Seong Young Ko, Jong-Oh Park, and Sukho Park (2012); Remote Controlled Micro-robots using Electromagnetic Actuation (EMA) Systems; The Fourth IEEE RAS/EMBS International Conference on Biomedical Robotics and Biomechatronics.

Karas Chris S., E. Antonio Chiocca (2007); Neurosurgical robotics: a review of brain and spine applications; Journal of Robotic Surgery; March 2007, Volume 1, Issue 1, pp 39-43.

Kim MinJun, Anak Agung Julius, U Kei Cheang (2017); Microbiorobotics, Biologically Inspired Microscale Robotic Systems, Elsevier.

Kummer Michael P., Jake J. Abbott, Bradley E. Kratochvil, Ruedi Borer, Ali Sengul and Bradley J. Nelson (2010); OctoMag: An Electromagnetic System for
5-DOF Wireless Micromanipulation; IEEE Transactions on Robotics, Vol. 26, No. 6.

Mani Menon (2002); Technique of Da Vinci RobotAssisted Anatomic Radical Prostatectomy; ELSEVIER SCIENCE INC.

McKesson; ROBOT-Rxm; (C) (2018) McKesson Corporation [En línea]. Disponible en: http://www.mckesson.com; [Accedido: 20-may2018]

Microbotmedical, (2017); "Virob, Life in motion"; C Microbot Medical Inc. [En línea]. Disponible en: http://www.microbotmedical.com/; [Accedido: 20-may-2018]

Moreno Sierra Jesús, Carlos Núñez Mora, $\mathbf{M}^{\mathrm{a}}$ Isabel Galante Romo, Sara Prieto Nogal, José López García Asenjo1, y Angel Silmi Moyano (2008).; Prostatectomía Radical Asistida por Robot Da Vinci®: Un Año de Experiencia en ll Hospital Clínico san Carlos; Laparoscopia y Robótica Arch. Esp. Urol., 61, 3 (385-396).

Nagy Zoltán, Raymond Oung, Jake J. Abbott, and Bradley J. Nelson (2008); Experimental Investigation of Magnetic Self-Assembly for Swallowable Modular Robots; IEEE/RSJ International Conference on Intelligent Robots and Systems.

Nathoo Narendra, M.D., Ph.D., M Cenk Çavuşoğlu, Ph.D., Michael A. Vogelbaum, M.D., Ph.D., Gene H. Barnett, M.D.(2005); In Touch with Robotics: Neurosurgery for the Future, Neurosurgery, Volume 56, Issue 3, 1 March 2005, Pages 421433.

Nelson Bradley J., Ioannis K. Kaliakatsos, and Jake J. Abbott (2010); Microrobots for Minimally Invasive Medicine; Institute of Robotics and Intelligent Systems, ETH Zurich, Zurich, Switzerland.

Nelson B. J., I. K. Kaliakatsos and J. J. Abbott, (2010); "Microrobots for Minimally Invasive Medicine," Annual Reviews, 2010.

Nguyen Van Du, Ji-Won Han, Young Jin Choi, Sunghoon Cho, Shaohui Zheng, Seong Young Ko, Jong-Oh Park, Sukho Park (2016); Active tumortherapeutic liposomal bacteriobot combining a drug (paclitaxel)-encapsulated liposome with targeting bacteria (Salmonella Typhimurium); Sensors and Actuators B, 217-224.

Osborne J.A. (2017); ForHEalth Technologies Inc, USA, IntelliFill iv; Disponible en: https://www.gerpac.eu/intellifill-i-v; [Accedido: 20-may-2018] 
Pandey Shilpa, Nitisha Payal,Aarti Sharma (2012); Robots and Robotically Assisted Surgeries; International Journal of Scientific Research Engineering \& Technology (IJSRET); Volume 1 Issue 5 pp 294298 August 2012

Park Sung Jun, Seung-Hwan Park, Sunghoon Cho, DeokMi Kim, Yeonkyung Lee, Seong Young Ko, Yeongjin Hong, Hyon E. Choy, Jung-Joon Min, Jong-Oh Park \& Sukho Park (2013); New paradigm for tumor theranostic methodology using bacteria-based microrobot; Nature Scientific Reports.

Park S., K. Cha and J. Park (2010) "Development of Biomedical Microrobot for Intravascular Therapy," International Journal of Advanced Robotic Systems.

Park Sukho, Kyoungrae Cha, and Jongoh Park; (2010) Development of Biomedical Microrobot for Intravascular Therapy; International Journal of Advanced Robotic Systems, Vol. 7, No. 1.

Pawashe Chytra, Steven Floyd, and Metin Sitti (2009); Multiple magnetic microrobot control using electrostatic anchoring; Applied Physics Letters 94.

Peyer Kathrin E., Li Zhang and Bradley J. Nelson (2013); Bio-inspired magnetic swimming microrobots for biomedical applications; Nanoscale, 5, 1259.

Purcell E. M. (1976); Life at Low Reynolds Number; Harvard University, Cambridge, Massachusetts.

Romagnuolo J., P. Cotton, G. Eisen, J. Vargo and B. Petersen (2011); "Identifying and reporting risk factors for adverse events in endoscopy".

Tewari Ashutosh, James Peabody, Richard Sarle, Guruswami Balakrishnan, Ashok Hemal, Alok Shrivastava, and

Villangca, Mark Jayson; Palima, Darwin; Banas, Andrew Rafael; Glückstad, Jesper (2016); Light-driven micro-tool equipped with a syringe function; Light: Science \& Applications; 2016.

Wautelet Michel (2001); Scaling laws in the macro-, micro- and nanoworlds; European Journal of Physics, Eur. J. Phys. 22, 601-611.

Yesin K. Berk, Philipp Exner, Karl Vollmers, and Bradley J. Nelson (2005); Design and Control of In-Vivo Magnetic Microrobots; Springer-Verlag Berlin Heidelberg.

Yim S. and M. Sitti (2012); "Design and Rolling Locomotion of a Magnetically Actuated Soft Capsule Endoscope,".
Yu Chungseon, Juhyun Kim, Hyunchul Choi, Jongho Choi, Semi Jeong, Kyoungrae Cha, Jong-oh Park, Sukho Park (2010); Novel electromagnetic actuation system for three-dimensional locomotion and drilling of intravascular microrobot; Sensors and Actuators A 161 (2010) 297-304.

Zhang Li, Jake J. Abbott, Lixin Dong, Kathrin E. Peyer, Bradley E. Kratochvil, Haixin Zhang, Christos Bergeles, and Bradley J. Nelson (2009); Characterizing the Swimming Properties of Artificial Bacterial Flagella; Nano Letters, Vol. 9, No. 10, 3663-3667. 